\title{
Proline synthesis in developing microspores is required for pollen development and fertility
}

\author{
Roberto Mattioli ${ }^{1}$, Marco Biancucci ${ }^{1,2}$, Amira El Shall ${ }^{1}$, Luciana Mosca ${ }^{3}$, Paolo Costantino ${ }^{1}$, Dietmar Funck ${ }^{4^{*}}$ (D) and \\ Maurizio Trovato ${ }^{1 *}$
}

\begin{abstract}
Background: In many plants, the amino acid proline is strongly accumulated in pollen and disruption of proline synthesis caused abortion of microspore development in Arabidopsis. So far, it was unclear whether local biosynthesis or transport of proline determines the success of fertile pollen development.

Results: We analyzed the expression pattern of the proline biosynthetic genes PYRROLINE-5-CARBOXYLATE SYNTHETASE 1 \& 2 (P5CS1 \& 2) in Arabidopsis anthers and both isoforms were strongly expressed in developing microspores and pollen grains but only inconsistently in surrounding sporophytic tissues. We introduced in a p5cs 1/p5cs 1 p5cs2/P5CS2 mutant background an additional copy of P5CS2 under the control of the Cauliflower Mosaic Virus (CaMV) 355 promoter, the tapetum-specific LIPID TRANSFER PROTEIN 12 (Ltp12) promoter or the pollen-specific At5g 17340 promoter to determine in which site proline biosynthesis can restore the fertility of proline-deficient microspores. The specificity of these promoters was confirmed by $\beta$-glucuronidase (GUS) analysis, and by direct proline measurement in pollen grains and stage-9/10 anthers. Expression of P5CS2 under control of the At5g17340 promoter fully rescued proline content and normal morphology and fertility of mutant pollen. In contrast, expression of P5CS2 driven by either the Ltp12 or CaMV35S promoter caused only partial restoration of pollen development with little effect on pollen fertility.

Conclusions: Overall, our results indicate that proline transport is not able to fulfill the demand of the cells of the male germ line. Pollen development and fertility depend on local proline biosynthesis during late stages of microspore development and in mature pollen grains.
\end{abstract}

Keywords: Proline biosynthesis, Pollen development, Tissue specificity, Anthers, Arabidopsis

\section{Background}

The importance of proline for pollen fertility has been recently highlighted by the observation that disruption of proline synthesis in Arabidopsis caused infertility by abortion during gametophyte development [1-3]. These findings provided a functional link between fertility and the accumulation of high levels of free proline under non-stressed conditions in floral organs - particularly

\footnotetext{
* Correspondence: dietmar.funck@uni-konstanz.de;

maurizio.trovato@uniroma1.it

${ }^{4}$ Department of Biology, University of Konstanz, Universitätsstraße 10, 78464 Konstanz, Germany

'Department of Biology and Biotechnology, Sapienza University of Rome, P.le A. Moro 5, 00185 Rome, Italy

Full list of author information is available at the end of the article
}

anthers and pollen grains - of different plant species [4-10]. However, it remained to be clarified whether this high level of proline in pollen is due to local synthesis or derives from import from other, sporophytic tissues.

In higher plants, proline is synthesized via a short pathway, which catalyzes the ATP- and NADPH-consuming reduction of glutamate to proline. In the first, rate-limiting step, glutamate is converted into glutamic semialdehyde by the bifunctional enzyme $\Delta^{1}$-pyrroline-5-carboxylate synthetase (P5CS). In the second step, glutamic semialdehyde spontaneously cyclizes to $\Delta^{1}$-pyrroline-5-carboxylate (P5C), which is further reduced to proline by the enzyme P5C reductase (P5CR). Some authors postulated an alternative route for proline synthesis from ornithine, catalyzed

(c) The Author(s). 2018 Open Access This article is distributed under the terms of the Creative Commons Attribution 4.0 International License (http://creativecommons.org/licenses/by/4.0/), which permits unrestricted use, distribution, and 
by the sequential actions of the enzymes ornithine- $\delta$-amino-transferase and P5CR [11, 12]. The differential localization of these two enzymes and the observation that functional P5CS expression is essential for reproduction (see below) suggested that synthesis from glutamate is the only functional pathway for proline biosynthesis in Arabidopsis $[2,3,13]$.

In Arabidopsis, P5CS is encoded by the two paralogous genes P5CS1 (At2g39800) and P5CS2 (At3g55610) [14]. P5CS1 is thought to be responsible for stress-induced proline accumulation, as homozygous $p 5 c s 1 \mathrm{mu}-$ tants did not accumulate proline under stress $[9,15]$. In contrast, P5CS2 is mostly involved in developmental processes such as embryo development and floral transition: $p 5 c s 2$ homozygous mutants are usually embryo lethal but can be rescued with external proline and can produce viable seeds under favorable conditions $[2,8$, 15]. Rescue of $p 5 c s 1 / p 5 c s 2$ double mutants has not been reported so far and quasi-double mutants homozygous for $p 5 c s 1$ and heterozygous for $p 5 c s 2$ ( $p 5 c s 1 / p 5 c s 1$ $p 5 c s 2 / P 5 C S 2$, from here on referred to as $p 5 c s$ sesquimutants) had very low levels of free proline in vegetative tissue, were late-flowering and showed reduced male fertility. In anthers of $p 5 c s$ sesquimutant plants, about half of the pollen grains are small, shriveled, devoid of nuclei and non-viable, as judged by Alexander staining [3]. When $p 5 c s$ sesquimutants were allowed to self-fertilize, the transmission of the $p 5 c s 2$ mutant allele to the next generation was lower than expected and formation of abortive homozygous $p 5 c s 2$ mutant embryos was not observed. Transmission of the $p 5 c s 2$ mutant allele from pollen of $p 5 c s$ sesquimutants to wildtype pistils was almost never observed ( 0 to $0.8 \%$ of observed transmission against $50 \%$ of expected transmission) indicating that only pollen with a functional P5CS allele developed normally and was fertile $[2,3]$.

Development of the male germline has been characterized in detail and divided into 13 stages [16]: Stages 1 to 7 comprise the development of sporogenous cells, meiosis and the release of free microspore tetrads inside anther locules. In stages 8 and 9 individual microspores are released from callose-encased tetrads and become vacuolated. From stage 10 onwards, both the microspore and the tapetum cells contribute to the formation of the pollen wall and exine. Stage 11 and 12 are marked by mitotic divisions giving rise to bi- and tri-cellular pollen, respectively. Stage 12 also prepares the release of the pollen grains by septum degeneration, which is completed in stage 13 with stomium rupture. In early stages, the sporogenous cells and the surrounding tapetum cells are interconnected by plasmodesmata, allowing the symplastic import of nutrients and macromolecules into the pollen mother cells [17]. The tapetum plays an essential role in pollen development and fertility as first demonstrated by genetic ablation of the tapetal cells in tobacco, which resulted in male sterility [18]. Later, Yang et al. (2003) [19] showed that a knock-out mutation of TAPETUM DETERMINANT 1 causes male-sterility in Arabidopsis by forcing tapetal cell precursors to differentiate into microsporocytes instead of tapetum. More recently, an essential function in the development of fertile pollen has also been demonstrated for the middle layer of the anther wall [20]. During meiosis, a thick callose wall is formed between and around the microspore tetrads, which become symplasmically isolated at this stage $[17,21]$. Accordingly, microspores depend on either apoplastic transport or endogenous synthesis for further accumulation of metabolites such as proline.

Available data suggest, but not demonstrate, that proline can be either synthesized in pollen grains or in surrounding sporophytic tissues. Indeed, microarray data indicate that all the genes involved in proline synthesis are expressed in developing Arabidopsis microspores and pollen grains as well as in anthers and flower buds $[22,23]$. Unfortunately, most microarrays used to analyze different parts of flowers do not distinguish between P5CS1 and P5CS2. We found one experiment (Gene Expression Omnibus accession GSM159352), in which gene-specific probes on a CATMA array yielded signals 2- to 10-times above background in both Col-0 and Ws-0 pollen [22, 24]. Székely et al. (2008) detected a P5CS1:GFP fusion protein in the pollen of Arabidopsis, an observation compatible either with transcription of P5CS1 in the male germline or with the import of P5CS1 protein or mRNA. The signal of P5CS2:GFP in anthers was less clear and appeared stronger in sporophytic anther tissues [15].

On the other hand, circumstantial evidence suggested that proline can also be actively transported from distant tissues or can be released by sporophytic anther tissues to be subsequently imported into pollen grains. The long distance transport of proline through phloem vessels from vegetative to floral tissues has been documented [25, 26] and the gene PROLINE TRANSPORTER 1 (ProT1; At2g39890) coding for a specific proline carrier in Arabidopsis has been shown to be highly expressed in mature pollen [27], both evidences apparently suggesting transport as the cause of accumulation of proline in pollen grains. However, none of the single, double and triple knock-out mutants of the genes belonging to the ProT family (At2g39890, At3g55740, At2g36590) exhibited alterations either in proline content or in pollen germination efficiency [27]. Expression of further amino acid carriers with broader substrate specificity, such as LYSINE HISTIDINE TRANSPORTER 2 (LHT2; At1g24400) and LHT4 (At1g47670), was detected in developing or mature pollen, but there is currently no information about the physiological function of these transporters in pollen [28]. 
To assess whether, and to which extent, the proline required for pollen fertility is locally synthesized in the male germ line or comes from surrounding sporophytic tissues, we analyzed the pattern of expression of P5CS1 and P5CS2 in Arabidopsis anthers, and generated and characterized $p 5 c s$ sesquimutant plants expressing a functional copy of P5CS2 either in vegetative tissue, in the tapetum or in developing pollen grains. Analyses of these plants show that the proline necessary for pollen development and particularly pollen fertility is mainly synthesized within developing microspores and mature pollen grains with little or no contribution from proline synthesized in the tapetum or other sporophytic tissues of the plant.

\section{Results}

The promoters of P5CS1 and P5CS2 are active in microspores and pollen grains at late stages of pollen development

As a first step to establish where the proline required for pollen development and fertility is synthesized, we analyzed the pattern of GUS activity under the control of the P5CS1 and P5CS2 promoter regions in Arabidopsis anthers at different stages of development (Fig. 1, Additional file 1: Figure S1). As promoter sequences, we used 2932 bp upstream of the P5CS1 and $2097 \mathrm{bp}$ upstream of the P5CS2 start codon, according to Ábrahám et al. (2003) [29]. We selected and analyzed 18 independent transformants with
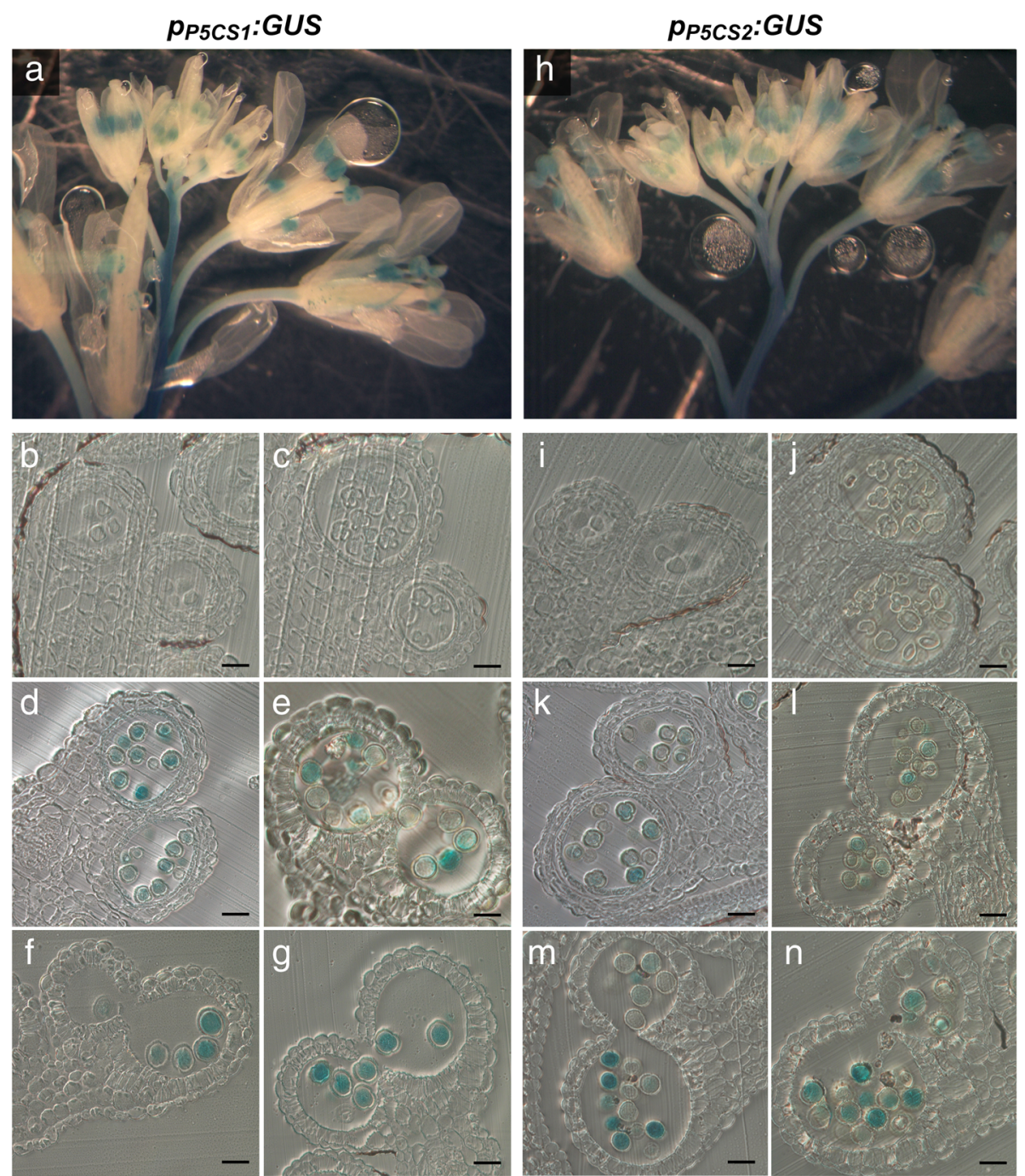

Fig. 1 Histochemical localization of GUS activity in anthers of $p_{P 5 C S 1}: G U S$ and $p_{P 5 C S 2}: G U S$ transgenic Arabidopsis. Inflorescences of $p_{P 5 C S 1}: G U S$ (a-g) and $p_{P 5 C s 2}$ GUS (h-n) transgenic plants were infiltrated with X-Gluc solution, stained overnight, fixed and cleared for microscopic analysis. $\mathbf{a}, \mathbf{h}$ Whole-mount inflorescences with buds and flowers at various developmental stages showing GUS activity almost exclusively in anthers. $\mathbf{b}-\mathbf{g}$ and $\mathbf{i}-\mathbf{n}$ Transverse sections of anthers at different developmental stages: $\mathbf{b}, \mathbf{i}$ Stage 8 with pre-meiotic microspore mother cells; $\mathbf{c}, \mathbf{j}$ Stage 9 with microspore tetrads; $\mathbf{d}, \mathbf{k}$ Stage 10 with strong activity of both pp5CS: GUS and pp5Cs: GUS after tetrad separation; e, I Stage 11 with fully developed exine and degenerating tapetum; $\mathbf{f}, \mathbf{m}$ Stage 12 with septum degeneration and $\mathbf{g}, \mathbf{n}$ stage 13 with mature pollen at anther dehiscence. Almost no GUS staining was detected in any sporophytic anther tissue. All scale bars $=20 \mu \mathrm{m}$ 
the $p_{P 5 C S 1}: G U S$ construct and 21 with $p_{P 5 C S 2}: G U S$. From each construct, we selected three homozygous lines with representative staining patterns for a more detailed analysis. Both $p_{P 5 C S 1}: G U S$ and $p_{P 5 C S 2}: G U S$ induced specific GUS expression in anthers, in the main stem and to a lesser extend in pedicels, but not in other parts of flowers or buds (Fig. 1a and h). Analysis of histological cross sections revealed that $p_{P 5 C S 1}: G U S$ and $p_{P 5 C S 2}: G U S$ were specifically active in developing microspores and mature pollen grains starting from stage 10 to stage 13 of pollen development, i.e. from immediately before the first mitotic division until the release of mature pollen (Fig. 1b, c, d, e, $f$ and $\mathrm{g}, \mathrm{i}, \mathrm{j}, \mathrm{k}, \mathrm{l}, \mathrm{m}$ and $\mathrm{n}$ ). Expression of either fusion construct was not detected in microspores at earlier stages of development (Fig. 1b and c, i and j), or in any sporophytic tissues of the anther at any stage (Fig. 1d, and k). These results suggest that in Arabidopsis anthers proline synthesis occurs almost exclusively in male gametophytic tissues, namely microspores and pollen grains starting from stage 10 , with little or no expression in surrounding sporophytic tissues, such as tapetum, middle layer and endothecium.

Bioinformatic promoter analysis, including gene ontology (GO) enrichment analysis, revealed that in the promoters of P5CS2 and, to a lesser extent, P5CS1 putative cis-regulatory elements are enriched for binding sites of transcription factors related to pollen development and fertility, pollen tube growth, anther development and double fertilization forming a zygote and endosperm (Additional file 2: Figure S2). In addition, the promoters of both P5CS1 and P5CS2 contain recognition sites for WRKY34 and WRKY2, well characterized transcription factors involved in pollen development and function [30, 31].

\section{CaMV35S promoter-driven sporophytic expression of P5CS2 does not rescue the abnormalities of $p 5 c s 1 / p 5 c s 2$ pollen}

In an attempt to complement the aberrant pollen development of $p 5 c s$ sesquimutants by overexpression of P5CS2, we introduced a transgenic copy of P5CS2 under the control of the CaMV35S promoter, which was reported to drive gene expression in most tissues and organs of Arabidopsis, although not in developing microspores and pollen grains [32]. To confirm the tissue-specificity of the CaMV $35 S$ promoter, we analyzed GUS activity in developing anthers of plants carrying a $p_{35 s}:$ GUS construct by histochemical staining. In whole inflorescences $p_{35 s}$ :GUS induced diffuse GUS activity in pistils, filaments and sepals as well as in floral buds at different developmental stages (Fig. 2a, Additional file 3: Figure S3A,B). Histological cross sections of anthers from $p_{355}$ :GUS showed strong GUS activity in vascular bundles (Fig. 2c) and light and diffuse activity of the CaMV35S promoter in all sporophytic tissues of the anther and in microspores at stage 8 , soon after meiotic division (Fig. 2b, c and d). At stage 9, in uninucleate microspores and in pollen grains of stage 11 and 12, no GUS activity was detected (Fig. 2e and f), confirming the results of Wilkinson et al. (1997) [32].

Subsequently, a $p_{35 s}:$ P5CS2 construct was introduced into $p 5 c s$ sesquimutants to evaluate the effects of the constitutive expression of P5CS2 in parental sporophytic tissues on the development of $p 5 c s 1 / p 5 c s 2$ double mutant pollen. However, in spite of the presence of the $p_{355}:$ P5CS2 construct, we detected low levels of free proline in inflorescences (Fig. 3a, Additional file 4: Table S1). High levels of P5CS2 expression and proline accumulation were observed during early stages of vegetative plant development, up to pre-flowering stage, while, in subsequent stages, both P5CS2 expression and the level of free proline dropped to levels similar to the original $p 5 c s$ sesquimutant line, indicating that the $p_{35 s: P 5 C S 2}$ transgene was silenced (data not shown). In a previous study, we observed that mild salt stress abrogated co-suppression of both endogenous P5CS genes induced by a transgenic $p_{35 s}:$ P5CS1 construct [9]. Accordingly, salt treatment $(0.1 \mathrm{M} \mathrm{NaCl})$ of $p 5 c s$ sesquimutants carrying the $p_{355}: P 5 C S 2$ construct restored proline synthesis to a level similar to non-stressed wildtype plants (Fig. 3a, Additional file 4: Table S1). However, the pattern of GUS activity induced by the $p_{35 s}$ :GUS construct in anthers was not altered by treatment with $0.1 \mathrm{M} \mathrm{NaCl}$ (data not shown).

Both under normal conditions or under mild salt stress treatment, no statistically significant differences were noticed between $p 5 c s$ sesquimutant plants with or without the $p_{355}: P 5 C S 2$ transgene, neither in the frequency of malformed pollen (Fig. 3b, c, d, e, f, g and h) nor in the proportion of aborted embryos in siliques of self-pollinated plants (Fig. 3i, j, k, l, m and n). Furthermore, the presence of the additional $p_{35 s}:$ P $5 C S 2$ transgene did not enable the transmission of the $p 5 c s 2-1$ mutant allele to the F1 generation when wildtype pistils were cross-pollinated with $p 5 c s$ sesquimutant pollen (Additional file 4: Table S1). It is important to notice that in spite of the identical pollen phenotype (i.e. high frequency of aberrant pollen grains and no transmission of the $p 5 c s 2-1$ allele), $\mathrm{NaCl}$ treatment induced a significant increase of free proline levels in anthers of $p 5 c s$ sesquimutant plants when the $p_{35 s}:$ P5CS2 transgene was present (Fig. 3a). These findings indicate that free proline in sporophytic anther tissues at a level similar to non-stressed wildtype plants is not sufficient to complement the developmental defects of $p 5 c s 1 / p 5 c s 2$ double mutant pollen.

\section{Selection of tapetum- or microspore-specific promoters}

To further investigate the effect of proline synthesis in the tapetum - the layer of sporophytic cells closest to 

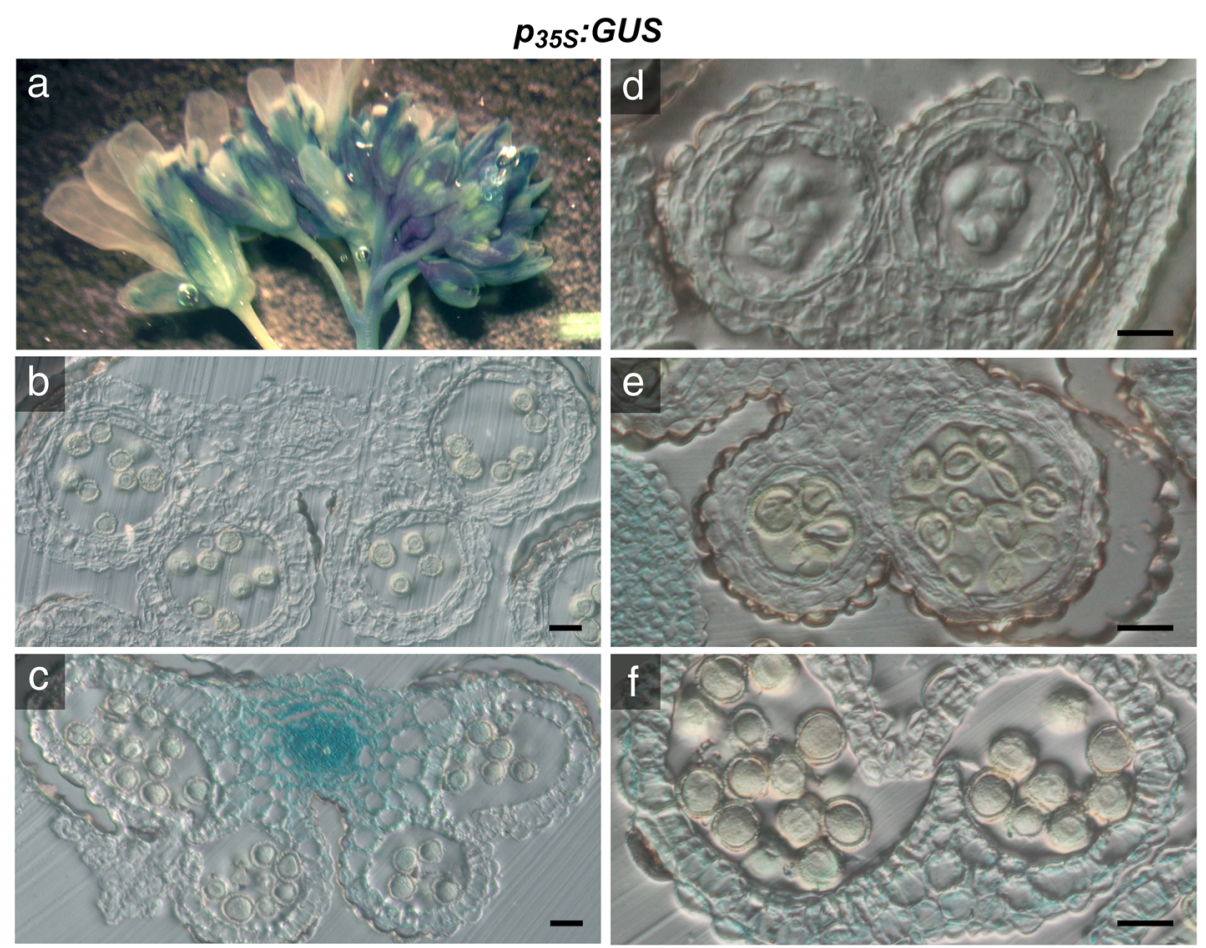

Fig. 2 Histochemical localization of GUS activity in anthers of $p_{35}$ : GUS transgenic Arabidopsis plants. Inflorescences of $p_{35}$ : GUS transgenic plants were infiltrated with X-Gluc solution, stained overnight, fixed and cleared for microscopic analysis. a Whole inflorescences of $p_{355}$ :GUS with GUS staining in filaments, sepals, pistils and in floral buds at different developmental stages. b-f Histological cross sections of $p_{355}: G U S$ anthers: $\mathbf{b}$ unstained anther at stage 11/12 showing faint blue iridescence due to light scattering. c Strong GUS staining at the vascular bundle at stage 11/12. No GUS activity was detected in fully mature pollen inside the pollen sacs. Bars in (b) and $(\mathbf{c})=50 \mu \mathrm{m}$. $\mathbf{d}$ Stage 8 anther at a higher magnification showing weak, diffuse GUS staining in all sporophytic tissues. e Stage 9 anther with no GUS staining in uninucleate microspores. f Stage 12 anther with weak GUS activity in degenerating anther wall but not in mature pollen. The faint greenish color visible in some pollen grains might indicate residual activity of the 355 promoter, but could also derive from diffusion of the soluble intermediate of X-Gluc staining or a low intrinsic GUS-like activity typical of pollen grains [57-59]. Bars $=20 \mu \mathrm{m}$ in (d-f)

the anther locule -, and in microspores and pollen grains, we decided to target P5CS2 to these cells by the use of tissue-specific promoters. Based on available microarray and literature data, we chose the microsporeand pollen-specific promoter of At5g17340 ( $\left.p_{17340}\right)$ and the tapetum-specific Ltp12 (At3g51590) promoter [33$35]$. The specificity of these promoters was validated by generating Arabidopsis lines harboring $p_{17340}: G U S$ and $p_{\text {Ltp 12: }}$ GUS transcriptional fusion constructs and analyzing the pattern of GUS activity in anthers at different stages of development (Fig. 4, Additional file 3: Figure S3C-F). Confirming previous observations, $p_{\text {Ltp12: }}$ GUS induced strong GUS activity specifically in the tapetum in stages 9 and 10 of anther development, and GUS expression disappeared when the tapetum degenerated. At the same developmental stage, $p_{17340}: G U S$ induced strong GUS activity in microspores, which became even stronger in later stages of gametophytic development. Hardly any GUS activity was detected in other tissues of the anthers.
Tapetum-specific expression of P5CS2 reduces the incidence of aberrant pollen but leads to poor recovery of fertility of $p 5 c s 1 / p 5 c s 2$ pollen

Tapetum cells provide the nutrients for microspore development and contribute to the formation of the outer layers of the pollen coat [36]. To investigate if P5CS2 expression in the tapetum can substitute for proline synthesis in developing microspores, we introduced a $p_{\text {Ltp 12:P5CS2 }}$ construct into $p 5 c s$ sesquimutant plants. We selected more than 20 primary transformants that carried both the p5cs2-1 mutant allele and the $p_{\text {Ltp 12: }}$ P5CS2 transgene, of which nine carried a single copy of the transgene. In heterozygous plants, the proportion of malformed pollen was reduced compared to $p 5 c s$ sesquimutant plants, although to a variable degree (data not shown). We selected three homozygous lines representing moderate $\left(p_{L t p 12: P 5 C S 2^{m}}\right)$ and strong $\left(p_{\text {Ltp 12:P5CS2 }}\right)$ complementation of the pollen development defect of the $p 5 c s$ sesquimutant for more detailed analyses (Additional file 4: Table S1). The proportion of 

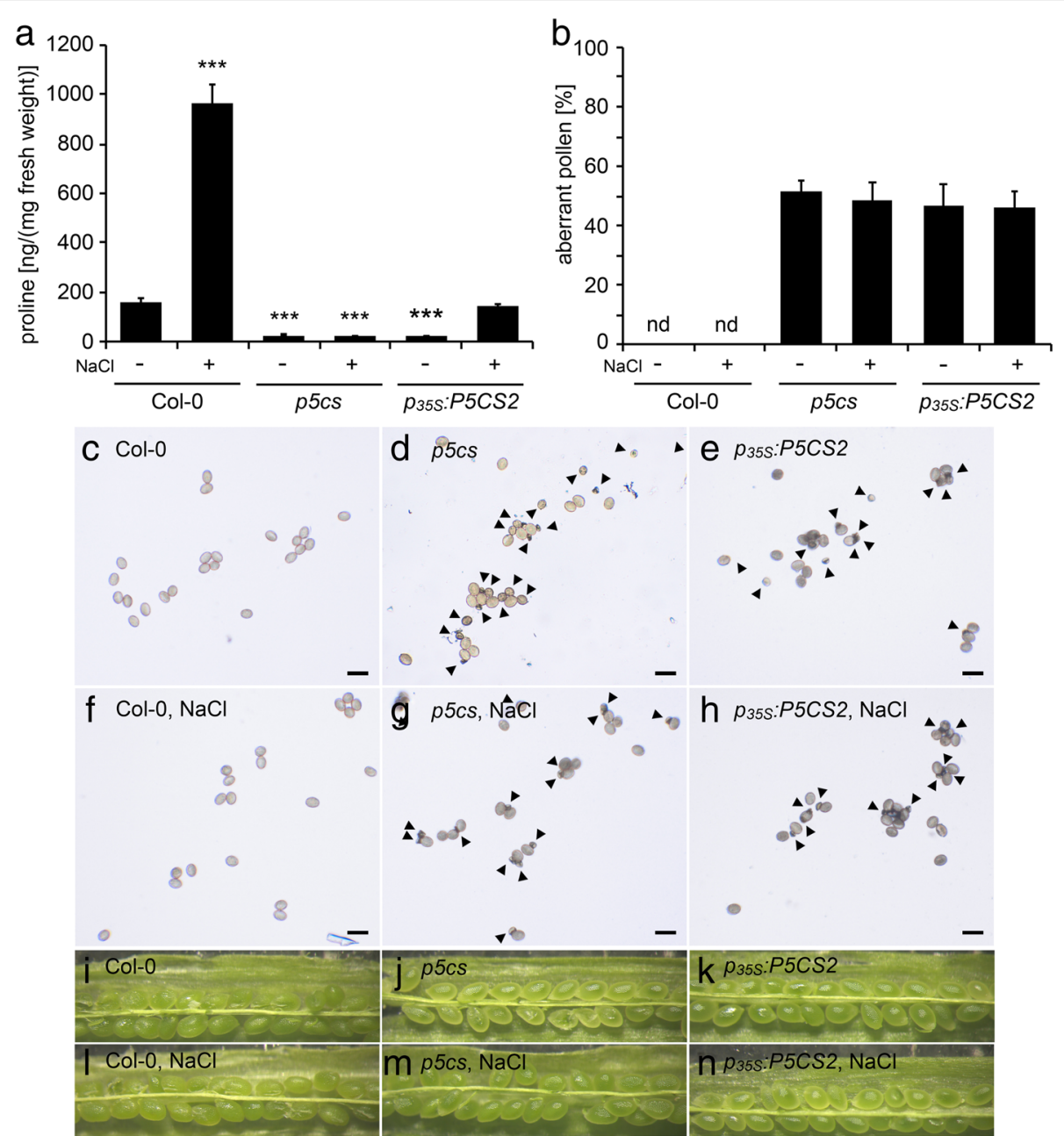

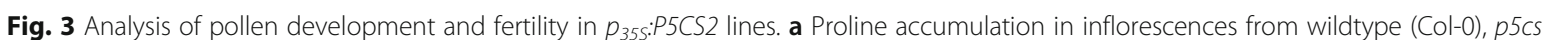
sesquimutants $(p 5 c s)$ and $p 5 c s$ sesquimutants homozygous for the $p_{355}$ :P5CS2 construct ( $p_{355}:$ P5CS2). Plants were either watered normally or exposed to $100 \mathrm{mM} \mathrm{NaCl}$, according to Material and Methods. Bars represent the mean $\pm \mathrm{SE}$ of at least three samples from different plants. ${ }^{* * *}$ indicates significant differences from Col-0 wildtype $(p<0.001$, by student's T-test). $\mathbf{b}$ Percentages of aberrant pollen grains in flowers from plants treated in the same way as in (a). Bars represent the means of \pm SE of 52 to 311 analyzed pollen grains from at least three independent plants. nd: not detected. $\mathbf{c}-\mathbf{h}$ Bright-field microscopic pictures of pollen grains from wildtype (c, $\mathbf{f}), p 5 c s(\mathbf{d}, \mathbf{g})$, and $p_{355}$ :P5CS2 (e, $\mathbf{h}$ ) lines either from normally watered plants $(\mathbf{c}, \mathbf{d}, \mathbf{e})$ or from plants treated with $\mathrm{NaCl}(\mathbf{f}, \mathbf{g}, \mathbf{h})$. Arrowheads indicate small, aberrant pollen grains. Bars $=50 \mu \mathrm{m}$. i-n Stereomicroscopic images of opened, premature siliques. No aberrant seeds, indicative of lethality of p5cs2-1 homozygous embryos (compare with Fig. 6), are visible inside the siliques, neither under normal conditions (i-k) nor after $\mathrm{NaCl}$ treatment (I-m)

aberrant pollen was $46 \pm 3 \%$ in $p 5 c s$ sesquimutant flowers, and this number was reduced to $31 \pm 1 \%$ and $18 \pm 2 \%$ in the $p_{L t p 12}: P 5 C S 2^{m}$ and the $p_{L t p 12}: P 5 C S 2^{s}$ lines, respectively (Fig. 5a).

Next, we determined the percentage of abortive em-

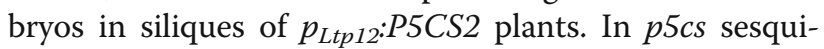
mutant plants, embryo-lethal homozygous individuals cannot be formed because of the infertility of pollen grains bearing the $p 5 c s 2-1$ allele, and therefore the siliques are nearly devoid of abortive embryos. In case of effective complementation of pollen fertility, $50 \%$ pollen grains bearing the $p 5 c s 2-1$ mutation would give rise to $25 \%$ homozygous $p 5 c s 1 / p 5 c s 2$ double mutants, which will be embryo-lethal because the $p_{L t p 12}: P 5 C S 2$ does not confer P5CS expression in embryos.

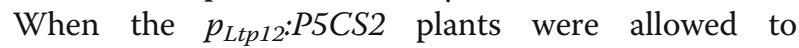
self-fertilize, the number of seeds that were aborted due to embryo development failures increased from $0 \%$ in $p 5 c s$ sesquimutant plants to $5 \pm 1 \%$ in the $p_{\text {Ltp } 12: P 5 C S} 2^{m}$ line and $6 \pm 1 \%$ in the $p_{L t p 12}: P 5 C S 2^{s}$ lines (Fig. $5 b$ ). In crosses with wildtype pistils, pollen from $p_{\text {Ltp12 }}: \mathrm{P} \mathrm{CS} 2^{\mathrm{m}}$ and $p_{L t p 12}: P 5 C S 2^{s}$ plants transmitted the $p 5 c s 2-1$ mutant allele to the next generation with a frequency of approximately $15 \%$ in all three $p_{L t p 12}: P 5 C S 2$ lines, which was far below the $50 \%$ expected for full complementation of the defects of $p 5 c s 1 / p 5 c s 2$ double mutant pollen (Fig. 5c). 

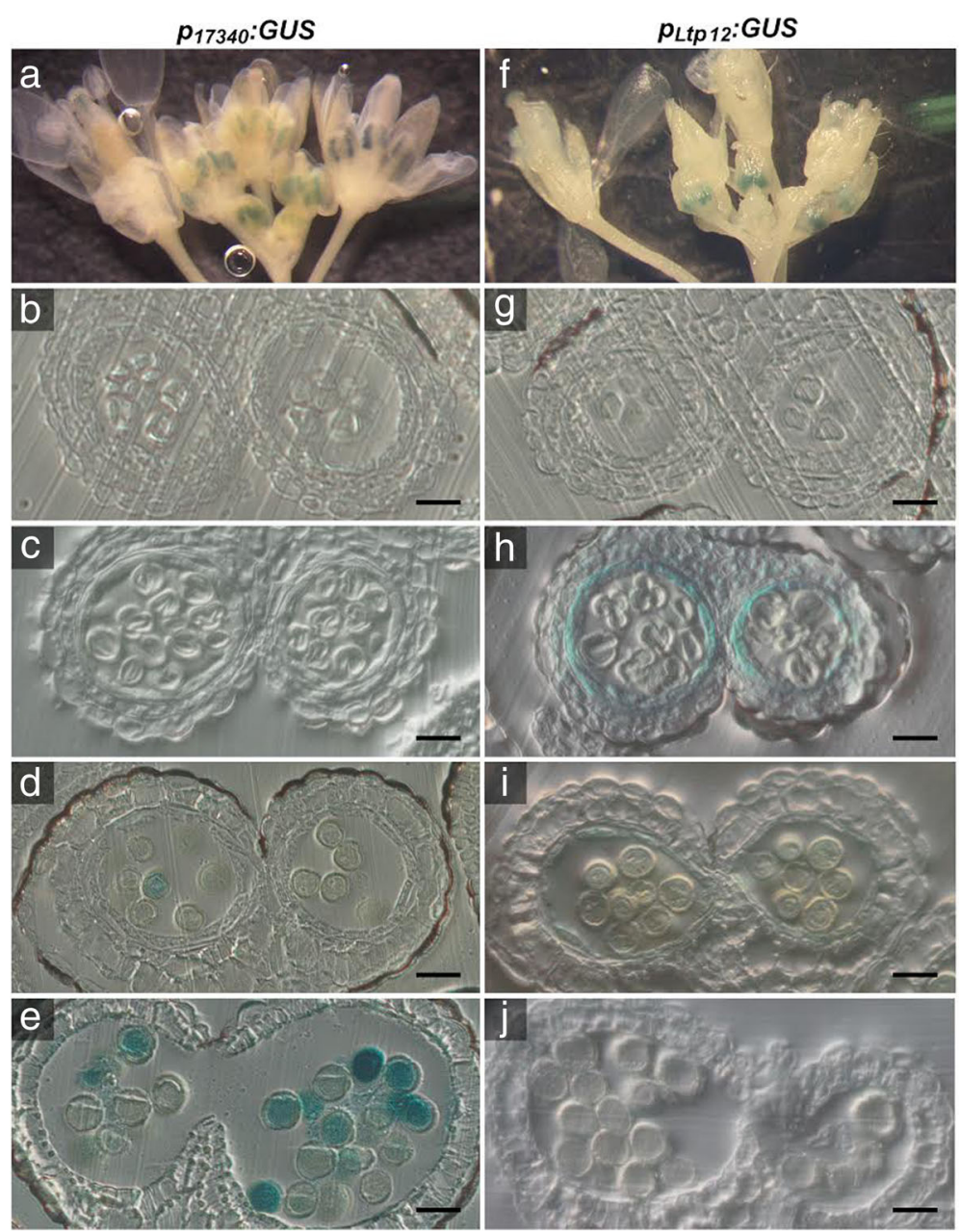

Fig. 4 Histochemical localization of GUS activity in anthers of $p_{17340: G U S}$ and $p_{L t p 12}: G U S$ transgenic Arabidopsis plants. Inflorescences of $p_{17340: G U S}$ $(\mathbf{a}-\mathbf{e})$ and $p_{\text {Ltp 12: }}$ GUS $(\mathbf{f}-\mathbf{j})$ transgenic plants were infiltrated with X-Gluc solution, stained overnight, fixed and cleared for microscopic analysis. $\mathbf{a}, \mathbf{f}$ Whole-mount inflorescences with buds and flowers at various developmental stages showing GUS activity exclusively in anthers. $\mathbf{b}$-e and $\mathbf{g}$-j Transverse sections of anthers at different developmental stages: $\mathbf{b}, \mathbf{g}$ Stage 8 with pre-meiotic microspore mother cells; $\mathbf{c}, \mathbf{h}$ Stage 9 with

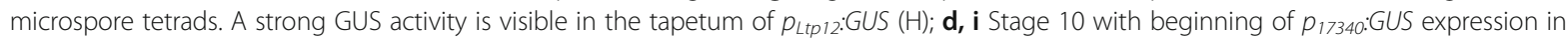

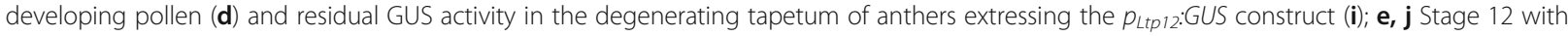

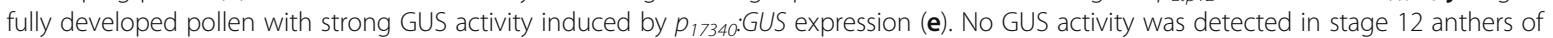

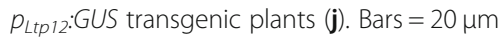

The observation of aborted embryos after selfing and the transmission of the $p 5 c s 2-1$ mutant allele via pollen in outcrossings indicated that P5CS2 expression in the tapetum was able to restore the fertility of $p 5 c s 1 / p 5 c s 2$ double mutant pollen, although only to a rather low degree.

\section{Pollen-specific expression of P5CS2 fully rescues the abnormalities of $p 5 c s 1 / p 5 c s 2$ pollen}

Histochemical GUS analysis of whole inflorescences and histological cross sections of anthers confirmed that the $p_{17340:}$ GUS was almost exclusively active in microspores and developing pollen from stage 10 of Arabidopsis anther development (Fig. 4, Additional file 3: Figure
S3E,F). Coherently, we generated a $p_{17340: P 5 C S 2}$ construct to target P5CS2 expression specifically to developing pollen and introduced it into $p 5 c s$ sesquimutant plants to verify if the pollen-specific expression of a functional P5CS2 could rescue the morphological and functional defects of $p 5 c s 1 / p 5 c s 2$ double mutant pollen grains. The expected results for an effective complementation would be a reduction in the number of aberrant pollen grains in the anther, and an increase in the number of abortive embryos in the silique - up to $25 \%$ for full complementation.

We selected 53 kanamycin resistant transformants and among them 16 that carried the $p 5 c s 2-1$ allele, which was similar to the transmission rate observed in 


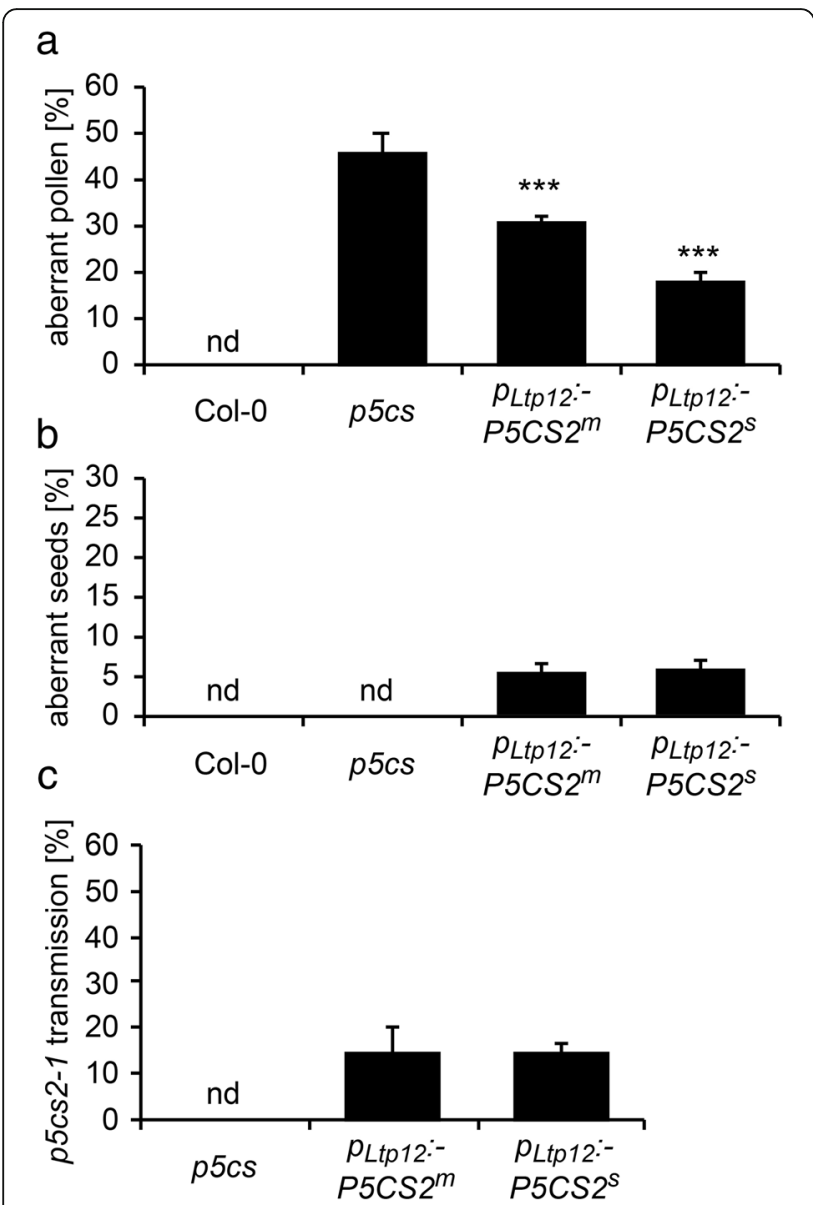

Fig. 5 Analysis of pollen development and fertility in $p 5 c s$ sesquimutants carrying the $p_{\text {Ltp 12: }}$ P5CS2 construct. a Percentages of aberrant pollen grains in wildtype (Col-0), $p 5 c s$ sesquimutants ( $p 5 c s)$ and $p 5 c s$ sesquimutants with a moderately $\left(p_{L t p 12: P 5 C S} 2^{m}\right)$ or strongly $\left(p_{\text {Ltp 12: }}:\right.$ 5 CS2 $\left.2^{5}\right)$ expressed $p_{\text {Ltp 12:P5CS2 construct. nd: not detected; *** }}$ indicate significant differences from $p 5 c s$ ( $p<0.001$, by student's T-test). b Percentages of aberrant seeds in siliques after self-fertilization of the genotypes described in a. c Percentages of p5cs2-1 mutant allelecarrying seedlings (scored by sulfadiazine resistance) obtained by cross-pollination of wildtype pistils with pollen from the plants described in $\mathbf{a}$. Bars in $\mathbf{a}, \mathbf{b}$ and $\mathbf{c}$ represent the mean \pm SE of, at least, three independent experiments. nd: not detected. Data on $p_{\text {Ltp 12: }}$ P5CS2 $2^{5}$ represents mixed data from two independent transgenic lines

non-complemented $p 5 c s$ sesquimutants. In anthers of the primary transformants, we observed between 5 and $20 \%$ aberrant pollen grains, indicating a copy-number dependent complementation by the $p_{17340}: P 5 C S 2$ construct. From transformants with a single-copy insertion of the $p_{17340: P 5 C S 2}$ construct, four independent homozygous lines were selected for further analyses.

In these batches of plants, the proportion of aberrant pollen was $43 \pm 1 \%$ in $p 5 c s$ sesquimutant plants and it was strongly reduced to $20 \pm 1 \%$ and $0.15 \pm 0.10 \%$ in heterozygous and homozygous $p_{17340}: P 5 C S 2$ transgenic plants, respectively (Fig. 6a, c, d, e and f, Additional file 4: Table S1). After self-pollination of $p_{17340: P 5 C S 2}$ plants, the frequency of aborted seeds in siliques with embryos at the bent cotyledon stage was $12 \pm 1 \%$ in heterozygous and 24 $\pm 2 \%$ in homozygous $p_{17340}:$ PCS2 plants (Fig. $6 \mathrm{~b}, \mathrm{~g}, \mathrm{~h}, \mathrm{i}$ and j). Appearance of $24 \pm 2 \%$ defective embryos in homozygous $p_{17340}: P 5 C S 2$ plants indicated that expression of $p_{17340: P 5 C S 2}$ restored the fertility of $p 5 c s 1 / p 5 c s 2$ double mutant pollen but was not able to restore embryo development of homozygous $p 5 c s 1 / p 5 c s 2$ double mutant embryos. Outcrossing to wildtype pistils confirmed that heterozygous $p_{17340: P 5 C S 2}$ plants produced $26 \pm$ $2 \%$ fertile pollen with a mutant $p 5 c s 2-1$ allele (48 sulfadiazine resistant plants out of 182 in total), which was increased to $46 \pm 1 \%$ when the pollen donor was homozygous for the $p_{17340: P 5 C S 2}$ construct (43 sulfadiazine resistant plants out of 94; Fig. 7, Additional file 4: Table S1).

\section{Recovery of pollen fertility correlates with proline concentration within microspores and pollen grains} To verify if tissue-specific expression of P5CS2 indeed resulted in altered levels of proline, we measured proline content in stage 9-10 anthers and mature pollen grains of wildtype, $p 5 c s$ sesquimutants, and $p 5 c s$ sesquimutants transgenic for the $p_{17340: P 5 C S 2} p_{\text {Ltp } 12: P 5 C S 2}$ or $p_{35 s: P 5 C S 2}$ constructs (Fig. 8a, Additional file 4: Table S1). Wildtype anthers contained $36 \pm 2 \mathrm{ng} / \mathrm{anther}$ free proline and for wildtype pollen, the proline content was calculated to be $39 \pm 2 \mathrm{pg} /$ grain. In anthers of $p 5 c s 2$ sesquimutant plants, the proline content was approximately one third $(12 \pm 2 \mathrm{ng} /$ anther) of the content in wildtype anthers. Averaged over normally developed and malformed pollen, the proline content in $p 5 c s 2$ sesquimutant pollen was $14 \pm 1 \mathrm{pg} /$ grain. Both $p_{\text {Ltp12:P5CS2 }}$ or $p_{355}:$ P5CS2 caused a significant increase in

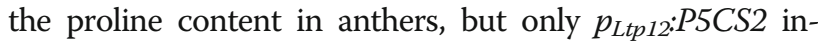
duced a higher proline content in pollen. Conversely, $p_{17340: P 5 C S 2}$ had no effect on the proline content in stage 9/10 anthers but restored the proline content in mature pollen to the level of wildtype pollen. When compared to the fertility of $p 5 c s 1 / p 5 c s 2$ double mutant pollen in the different lines, estimated from the appearance of embryo-lethal $p 5 c s 2-1$ homozygous seeds after selfing or from the transmission rate of the $p 5 c s 2-1$ allele to wildtype pistils, proline content of pollen showed a strong positive correlation $\left(R^{2}=0.98, p<0.001\right.$; Fig. $\left.8 b\right)$, whereas proline content in anthers did not correlate with pollen fertility (Fig. 8c).

\section{Discussion}

Consistent with the high proline concentration found in pollen grains of different plant species [4, 7, 10, 37], proline biosynthesis has been shown to be necessary for 

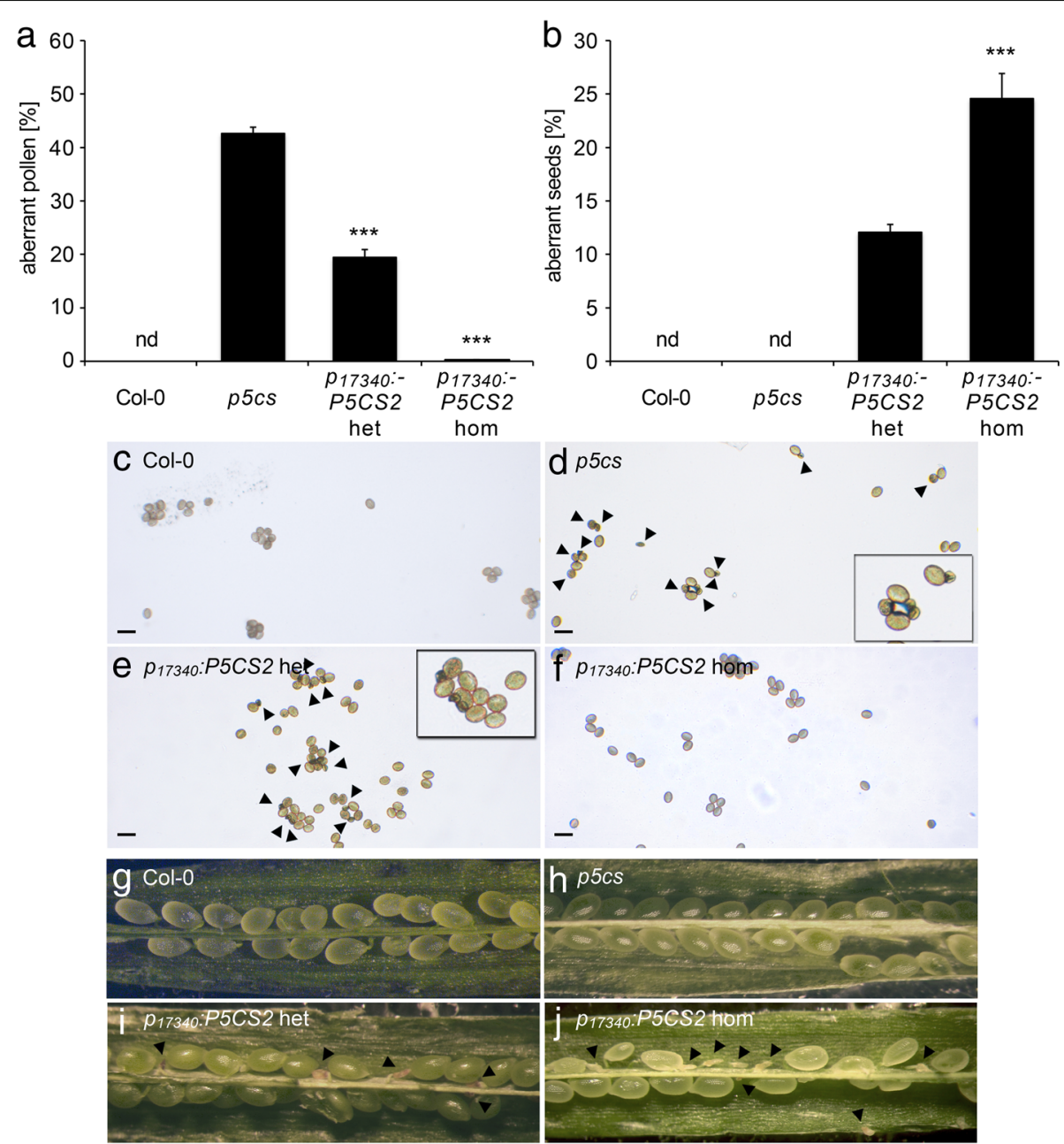

Fig. 6 Morphological analysis of pollen and seed defects in p5cs sesquimutants complemented by $p_{17340: P 5 C S 2}$ expression. a Percentages of aberrant pollen grains and $\mathbf{b}$ percentages of aberrant seeds in siliques from wildtype plants (Col-0), p5cs sesquimutants (p5cs) and heterozygous or homozygous complementation lines ( $p_{17340: P 5 C S 2}$ het/hom). Bars represent the means \pm SE of 16-22 samples per genotype in a and 9 siliques per genotype in $\mathbf{b}$ mixed from plants of two independent complementation lines. nd: not detected; In $\mathbf{a}{ }^{* * *}$ indicate significant differences from $p 5 C s$, while in $\mathbf{b}^{* * *}$ indicate significant differences between $p_{17340}:$ P5CS2 het and $p_{17340}:$ P5CS2 hom ( $p<0.001$, by student's T-test). Two further complementation lines produced similar results. c-f Bright-field microscopic pictures of pollen grains from wildtype $\mathbf{c}$ and $p 5 \mathrm{cs}$ sesquimutants with e-f or without $\mathbf{d}$ the $p_{17340}$ :P5CS construct in homozygous $\mathbf{f}$ or heterozygous state $\mathbf{e}$. An inset at higher magnification is also shown for the p5cs sesquimutant and the heterozygous complementation line. Arrowheads indicate small and shriveled pollen grains. $\mathbf{g}$-j Stereomicroscopic images of opened, premature siliques from wildtype $\mathbf{g}$ and $p 5 c s$ sesquimutants with $\mathbf{i}-\mathbf{j}$ or without $\mathbf{h}$ the $p_{17340: P 5 C S}$ construct. Aberrant seeds indicative of lethality of $p 5 c s 2-1$ homozygous embryos are indicated by arrowheads

pollen development and fertility in Arabidopsis [2, 3], but it was still unknown whether proline needs to be synthesized in developing pollen or can also be synthesized in sporophytic cells outside the pollen sac and transported into pollen grains.

\section{P5CS1 and P5CS2 are strongly expressed in developing microspores but not in sporophytic cells outside the pollen sac}

A first indication of the gametophytic origin of the proline accumulated in pollen grains derived from the analysis of the promoter activity of the proline biosynthetic genes P5CS1 and P5CS2 in Arabidopsis flowers. Although tissue-specific, development-related expression of P5CS1 and P5CS2 has already been reported [15, 38], a detailed histological analysis of the expression of these genes throughout anther development was still lacking. To fill this gap, we analyzed anthers of Arabidopsis lines expressing $p_{P 5 C S 1}: G U S$ or $p_{P 5 C S 2}: G U S$. Both constructs induced strong GUS expression in male gametophytic tissues but no significant expression in surrounding sporophytic tissues, which is partially in contrast to the data from Szekely et al. (2008), who observed green fluorescence induced by a $p_{P 5 C S 2}: P 5 C S 2: G F P$ construct 


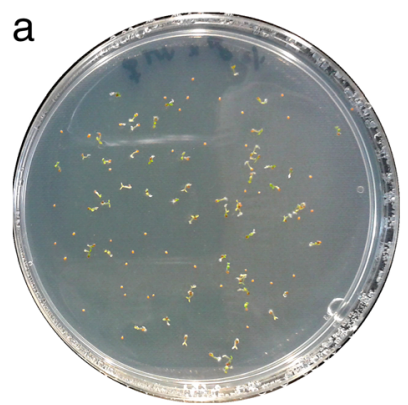

p5cs $\sigma^{\prime} \times$ Col-0

b

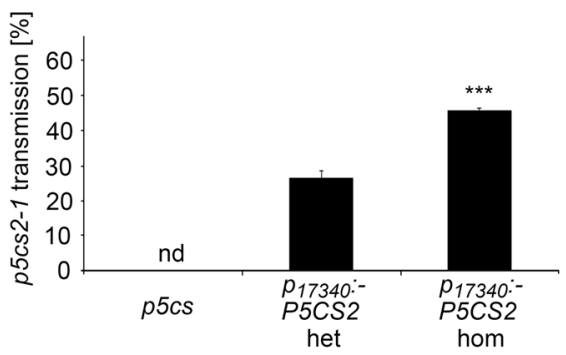

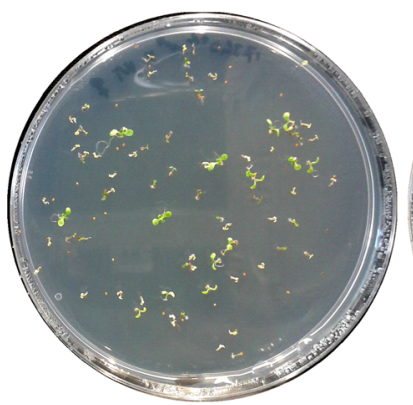

$p_{17340: P 5 C S 2}$ het $\sigma \times$ Col-0 $Q$

$\mathrm{C}$

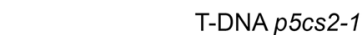

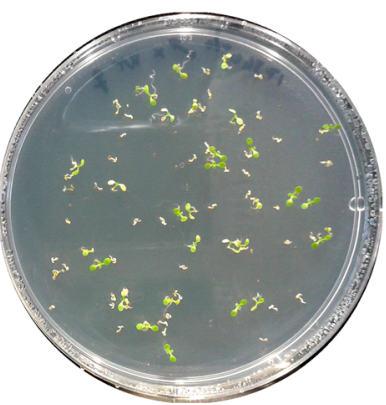

$p_{17340: P 5 C S 2 \text { hom } \sigma} \sigma$ Col-0 우

T-DNA $p 5 c s 2-1$

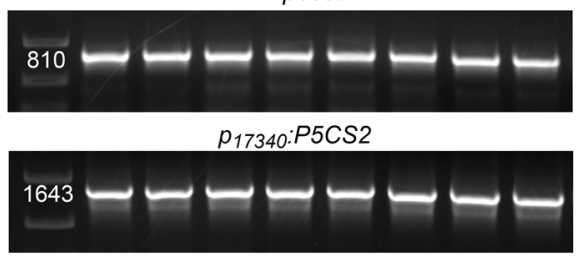

Fig. 7 Expression of $p_{17340}$ :P5CS2 restores fertility of $p 5 \operatorname{cs} 1 / p 5 \operatorname{cs} 2$ double mutant pollen. a Seeds produced by wildtype pistils fertilized with pollen from either a $p 5 c s$ sesquimutant (leftmost panel), or a $p 5 c s$ sesquimutant heterozygous (middle panel) or homozygous (rightmost panel) for the $p_{17340: P 5 C S 2}$ construct were germinated on sulfadiazine-containing plates to score for the transmission of the $p 5$ cs $2-1$ allele. $\mathbf{b}$ Percentages of sulfadiazine-resistant seedling among the progeny of wildtype pistils after cross-pollination as described in a Bars represent the mean \pm SE of, at least, three independent experiments with plants of two independent transgenic lines. nd: not detected; ${ }^{* * *}$ indicates significant difference to $p_{17340}$ :P5CS2 het ( $p<0.001$ by student's T-test). Two further lines produced very similar results. $\mathbf{c}$ PCR analysis of the sulfadiazine-resistant progeny. Individual resistant plantlets were analyzed by PCR for the simultaneous presence of both the T-DNA generating the $p 5 c s 2-1$ mutation (upper panel) and the $p_{17340}$ P5CS2 construct (bottom panel). The sizes of the PCR products matched the expected numbers of base pairs (indicated alongside)

in sporophytic anther tissue but only sporadically in pollen or precursor cells. Export of P5CS2 mRNA or protein from the gametophytic cell line into surrounding anther tissue is possible, but highly unlikely at later stages of pollen development. The strong GUS staining observed in microspores and pollen from both $p_{P 5 C S 1}: G U S$ and $p_{P 5 C S 2}: G U S$ plants indicates a strong activity of the P5CS1 and P5CS2 promoters, which is consistent with publicly available transcriptome data [22, 23, 35]. It is possible that the GUS mRNA or protein differs in stability from the Arabidopsis P5CS1 and P5CS2 proteins and thus may not entirely reflect the timing of P5CS1 and P5CS2 expression in the male germline. Proteome analyses of Arabidopsis microspores or pollen did so far not detect peptides derived from P5CS1 or P5CS2 [39-41]. However, in a recent study of tobacco pollen development, P5CS-derived peptides were detected in mature pollen and in pollen tubes but not at earlier developmental stages [42]. A metabolomic analysis of development of the tobacco male gametophyte found that proline accumulation started with pollen mitosis I and peaked in desiccated pollen, while proline concentration quickly declined during pollen tube growth [43]. In the same study, Rotsch et al. (2017) reported a similar pattern of accumulation also for pipecolic acid, a non-proteinogenic amino acid that differs from proline by one additional $\mathrm{CH}_{2}$-group in the ring structure.

In addition, bioinformatic identification of putative cisregulatory elements in the upstream regions of P5CS1 and P5CS2, which were analyzed with PlantPan 2.0 and Place, revealed an enrichment in putative binding sites for transcription factors related to pollen development and fertility. In particular, the promoter of P5CS2 contains binding motifs for WRKY2 and WRKY34, the best-characterized transcription factors involved in pollen development and function [30, 31]. No putative binding sites for transcription factors related to tapetum development or function were identified by these programs, indicating that our localization data obtained with the $p_{P 5 C S 2}: G U S$ construct is accurate.

\section{Only P5CS2 overexpression in microspores and pollen grains fully recovers the abnormalities of $p 5 c s 1 / p 5 c s 2$ double mutant pollen grains}

To confirm the importance of proline synthesis in gametophytic cells, we generated $p 5 c s$ sesquimutant plants containing an additional copy of P5CS2 expressed specifically either in gametophytic or sporophytic cells of the anther. The rationale was to assess which construct, 


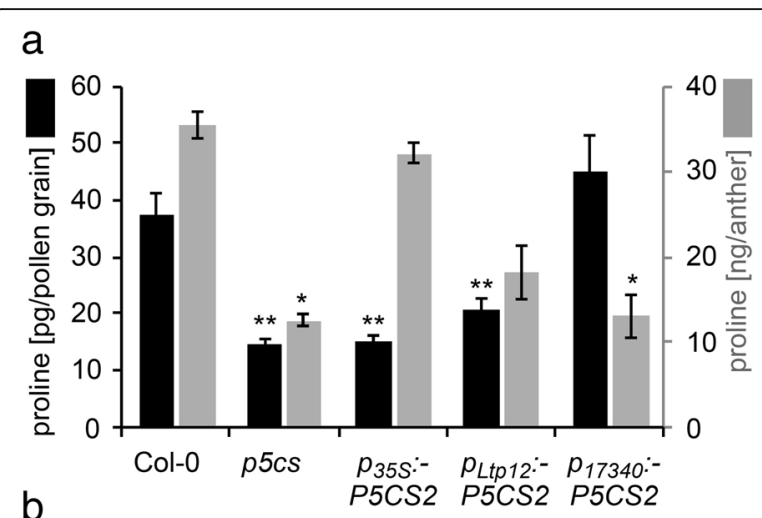

b

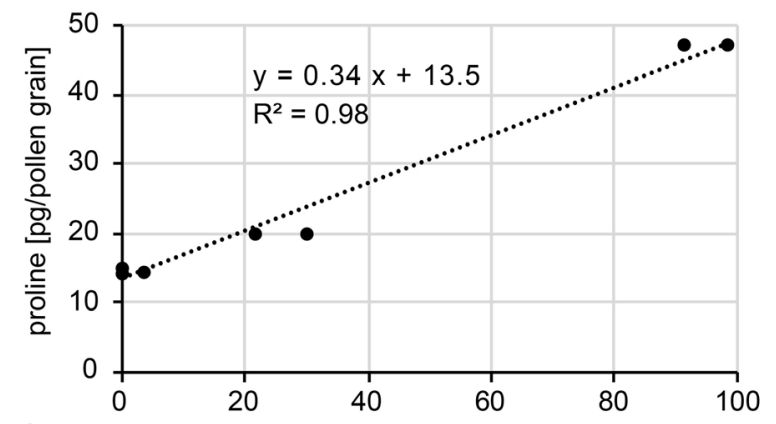

C

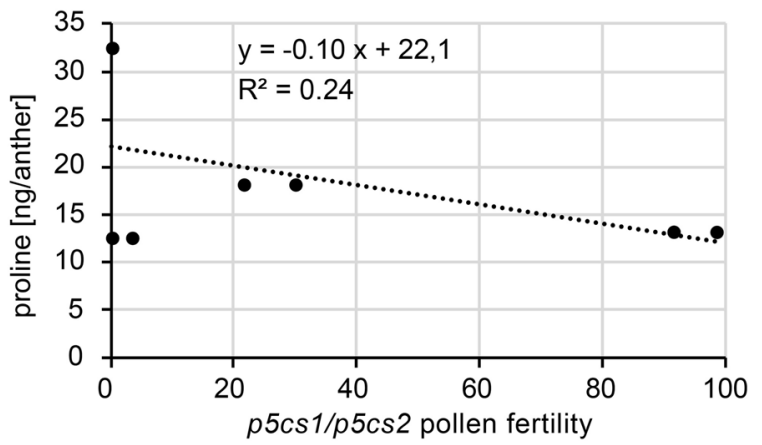

Fig. 8 Pollen fertility correlates with proline concentration in pollen grains. a Proline content in pollen grains (black bars, left axis) and stage $9 / 10$ anthers (grey bars, right axis) from wildtype (Col-0), p5cs sesquimutants ( $p 5 c s)$ and $p 5 c s$ sesquimutants carrying either the $p_{355}: P 5 C S 2$, the $p_{L t p 12}: P 5 C S 2^{m}$ or the $p_{17340}:$ P5CS2 construct. For every analysis an average of 1000 pollen grains or 200 anthers of stage 9-10 were collected and processed. Bars represent the mean \pm SE of two (anthers) or three (pollen) independent samples. * and ** indicate significant differences from the corresponding Col-0 wildtype samples ( $p<0.05$ or $p<0.01$, respectively, by student's T-test). $\mathbf{b}, \mathbf{c}$ Fertility of $p 5 c s 1 / p 5 c s 2$ double mutant pollen as estimated in Additional file 4: Table S1 in the different complementation lines was correlated either to the amount of proline in pollen grains $\mathbf{b}$ or to the amount of proline in anthers at stage 9-10 c. A strong correlation (dotted regression line) was found between proline accumulation in pollen grains and pollen fertility $\left(R^{2}=0.98, P<0.001\right)$, while no significant correlation (dotted regression line) was found between proline accumulation in anthers of stage $9-10$ and pollen fertility

and to what extent, could rescue the functionality of p5cs1/p5cs 2 double mutant pollen. We show that expression of $\mathrm{P} 5 \mathrm{CS} 2$ in microspores and pollen grains with the pollen-specific promoter of At5g17340 resulted in full complementation of the developmental and fertility defects of $p 5 c s 1 / p 5 c s 2$ double mutant pollen. We conclude that P5CS activity inside microspores and developing gametophytes is sufficient to provide the proline needed for pollen development and fertility.

Conversely, $p 5 c s$ sesquimutants expressing an additional

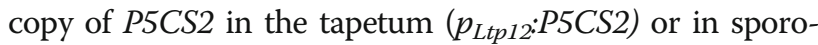
phytic cells outside the pollen sac $\left(p_{35 s}: P 5 C S 2\right)$ showed only very limited or no complementation of $p 5 c s 1 / p 5 c s 2$ double mutant pollen morphology and fertility. Expression

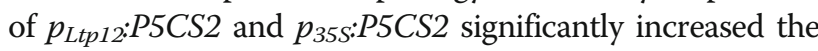
levels of proline in anthers but had little or no effect on the proline content in pollen grains of $p 5 c s$ sesquimutants. The observation that the $p_{\text {Ltp12: }}$ P5CS2 construct complemented the pollen abnormalities of $p 5 c s$ sesquimutants more efficiently than $p_{35 s}: P 5 C S 2$ in spite of the higher level of proline found in anthers of the latter may be explained

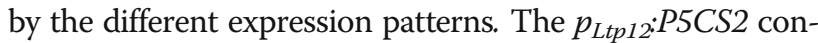
struct induces P5CS2 expression in the tapetum, which directly surrounds the cells of the gametophytic cell lineage. Obviously transport of proline to microspores is more efficient over short distances and might occur through plasmodesmata at early stages of microspore development or via proline leakage from tapetal cells into the extracellular space, potentially during tapetum degeneration. The capacity of developing pollen to take up extracellular proline by means of high ProT1 expression has been demonstrated, although the absence of pollen defects in proT mutants suggested that it is of minor importance under wildtype conditions [27]. In contrast, proline synthesized through the activity of $p_{35 s}: P 5 C S 2$ in the anther vasculature does not seem to be accessible for developing pollen.

The failure of P5CS2 expression in the tapetum or the anther vasculature to fully restore development and fertility of $p 5 c s 1 / p 5 c s 2$ double mutant pollen strongly indicates, that P5CS expression in the male germline is not only sufficient, but also necessary for pollen fertility. Consistently, a strong correlation between proline content in pollen and fertility was observed, whereas proline levels in anthers did not show a clear correlation with fertility of $p 5 c s 1 / p 5 c s 2$ double mutant pollen. It remains to be determined, which precursors are used for proline biosynthesis and how they are delivered to the developing pollen to ensure full fertility.

\section{Possible functions of proline accumulation in pollen}

Several functions have been proposed for the high accumulation of proline in pollen: It may serve to maintain pollen viability despite desiccation during the transport to distant pistils, it may help the pollen to rehydrate after the arrival at the pistil and it may provide an energy source or source of building material during pollen tube growth [1, 44]. Additionally, signaling functions 
have been proposed for proline as well as for the structurally similar pipecolic acid, whereas so far, no functional or metabolic link between these two metabolites has been reported [45-47]. Furthemore, proline may serve as a reward for pollen dispersing insects that often deliver only a small fraction of the pollen to other flowers and use the major part as energy- and protein-rich food source.

Our data demonstrate that high levels of proline are required already for the development of fertile pollen. The misshaped and infertile $p 5 c s 1 / p 5 c s 2$ double mutant pollen of $p 5 c s$ sesquimutants were shown to be devoid of DNA and storage compounds, indicating that a controlled cell death program is initiated during development [3]. It remains to be determined if programmed cell death is initiated specifically by the lack of proline or merely by developmental retardation of proline deficient microspores compared to proline synthesizing neighbors. In maize roots, the level of proline regulated cell cycle progression and a similar mechanism may be in place during gametophytic mitoses giving rise to trinucleate pollen [48]. P5CS2 expression in the tapetum having a stronger effect on development than on fertility of $p 5 c s 1 / p 5 c s 2$ double mutant pollen indicated that the threshold for cell death induction might be lower than the proline level required to support full fertility.

\section{Conclusions}

In this study, we show that most, if not all, of the effects of proline on pollen development and fertility can be accounted for by local synthesis inside developing microspores and mature pollen grains, and that the contribution of proline transport from different sporophytic tissues, if any, is very limited. Our findings open up interesting possibilities for breeding approaches: Overproduction of proline in pollen may increase pollen fertility and thus crop yield under adverse conditions, whereas inhibition of proline biosynthesis in pollen would enable conditional male sterility for hybrid production.

\section{Methods}

\section{Plant growth conditions}

Wildtype and mutant Arabidopsis thaliana (L) Heynh., ecotype Columbia-0 (Col-0), were grown in a growth chamber at $24 / 21^{\circ} \mathrm{C}$ with light intensity of $300 \mu \mathrm{E} \mathrm{m} \mathrm{m}^{-2}$ $\mathrm{s}^{-1}$ under $16 \mathrm{~h}$ light and $8 \mathrm{~h}$ dark per day. An Arabidopsis line homozygous for $p 5 c s 1$ and heterozygous for $p 5 c s 2$ (referred to as $p 5 c s$ sesquimutant), has been characterized and described previously $[2,3]$. To carry out salt treatment, plants were watered twice a week with $0.1 \mathrm{M} \mathrm{NaCl}$ solution from the onset of flowering to the end of the experiment. To analyze $p 5 c s 2-1$ mutant allele transmission via pollen during cross-pollination or during selfing, surface sterilized seeds were stratified for three days at $4{ }^{\circ} \mathrm{C}$, and germinated on $1 / 2 x M S$ plates supplemented with $12 \mu \mathrm{g} / \mathrm{ml}$ sulfadiazine and $2 \%(w / v)$ sucrose. Presence of the $p 5 c s 2-1$ mutant allele was further confirmed by PCR analysis of random samples using specific primers for the P5CS2:T-DNA junction or the sulfadiazine resistance gene (Additional file 5: Table S2).

\section{Generation of transgenic plants}

Molecular cloning techniques were performed according to standard protocols using primers listed in Additional file 5: Table S2. Enzymes used in this work were purchased from Thermo Fisher Scientific or New England Biolabs. RNA and DNA extractions were performed as previously described $[3,8]$. The constructs $p_{P 5 C S 1}: G U S$, $p_{P 5 C S 2}: G U S$ and $p_{17340}: G U S$ were generated by replacing the CaMV35S promoter in pBI121 (Clontech, Paolo Alto, California) with either the $2932 \mathrm{bp}$ upstream of the start codon of AtP5CS1, the $2097 \mathrm{bp}$ upstream of AtP5CS2 or the $2632 \mathrm{bp}$ upstream of At5g17340, either by direct ligation or by Gibson assembly. To generate $p_{355}:$ P5CS2 2 and $p_{17340}:$ P5CS2 constructs, the GUS coding sequence was replaced by the cDNA of AtP5CS2. For the $p_{L t p 12}: G U S$ construct, the first six codons of Ltp12 (At3g51590) and $1092 \mathrm{bp}$ of upstream sequence were inserted into pENTR-D-TOPO and transferred to pHGWFS7 by LR recombination [49]. For the $p_{\text {Ltp12:P5CS2 }}$ construct, the cDNA of AtP5CS2 and was inserted into pENTR-D-TOPO and the Ltp12 promoter region was inserted by Gibson assembly into a unique NcoI site generated at the start codon. The resulting $p_{\text {Ltp12:P5CS2 fusion }}$ construct was transferred into pEG301 by LR recombination [50]. All constructs including the native pBI121 were introduced into wildtype Arabidopsis plants or $p 5 c s$ sesquimutants by floral dip with Agrobacterium tumefaciens strain GV3101 [51]. Kanamycin, hygromycin or BASTA selection were used to isolate $\mathrm{T} 1$ transformants, T2 single-insertion lines, and T3 homozygous lines. Presence of the desired transgene in each line was confirmed by PCR and for each GUS or fertility analysis, homozygous plants from at least two independent lines were used.

\section{Assessment of pollen development and fertility}

To evaluate developmental aberrations, pollen grains were tapped on a glass slide, photographed under a light microscope and the percentage of aberrant pollen grains over total pollen grains was scored. Fertility was scored by comparing the expected inheritance rate during selfing, or in crosses of mutant pollen with wildtype pistils, with the observed transmission of the $p 5 c s 2-1$ mutant allele. For the analysis of embryo development, siliques were dissected under a stereomicroscope (Zeiss Stevi SV 6, Carl Zeiss Microimaging GmbH, Jena, Germany). Digital images were acquired with a Jenoptik ProgResW C3 digital camera (Jenoptik, Jena, Germany). All analyses have been 
repeated at least four times. Differences between expected and observed segregation or transmission ratios were analyzed for significance with $\chi^{2}$ tests.

\section{Proline analysis}

Proline content in seedlings or whole inflorescences was measured according to Bates [52], using L-proline as standard. Every measurement was repeated at least three times and represents the average either from more than one hundred 14-day-old seedlings or from inflorescences pooled from five plants. Proline content in anthers or pollen grains was measured by comparing HPLC chromatograms against an L-proline standard. To extract proline from pollen grains, about 10,000 pollen grains were collected from flowers on a glass slide. A representative number of microscopic sectors were photographed with an Axio Imager.A2 light microscope (Zeiss, Germany), equipped with a DC500 digital camera (Leica, Germany), and analyzed with ImageJ to estimate pollen number [53]. The pollen grains were collected from the glass slides in a suitable volume of $3 \%(w / v)$ 5 -sulfosalicylic acid $(\sim 50 \mu \mathrm{l})$ and subsequently concentrated under vacuum to adjust pollen concentration to $1,000 \mathrm{Hl}^{-1}$. To extract proline from anthers, about 200 anthers were collected at stage 9-10 under a dissection microscope and extracted in $50 \mu \mathrm{l}$ of $3 \% \quad(\mathrm{w} / \mathrm{v})$ 5-sulfosalicylic acid. The developmental stages of the anthers were established as described by Cecchetti et al. (2015) [54]. The extract was centrifuged at $14,000 \mathrm{~g}$ for 20 min and the supernatant reduced to $7 \mu \mathrm{l}$ under vacuum. The amino acids present in the extract were derivatized with DABS (4-N, N-dimethylaminoazobenzene-4' -sulfonyl chloride, Sigma-Aldrich, USA), according to Francioso et al. (2017) [55]. Gradient grade solvents used for chromatographic analyses were purchased from Carlo Erba Reagents (Milan, Italy). Amino acid standards and all other reagents were obtained from Sigma-Aldrich (St. Louis, MO, USA).

\section{Histochemical GUS staining and imaging}

For analysis of GUS activity, samples were infiltrated by vacuum for $1 \mathrm{~h}$ with 5-bromo-4-chloro-3-indolyl- $\beta$-D-glucuronide (X-Gluc) solution supplemented with $1 \mathrm{mM}$ potassium ferricyanide as an oxidative catalyst and then incubated over night at $37^{\circ} \mathrm{C}$ [56]. Green tissues were then fixed and cleared under constant agitation in methanol/ acetic acid (3:1, v:v) for $4 \mathrm{~h}$ at room temperature, followed by several washes in $70 \%$ ethanol. Plant samples for histological analysis were dehydrated, embedded in Technovit 7100 resin (Heräeus Kulzer, Wehrheim, Germany), and cut into $8 \mu \mathrm{m}$ sections with a HM $350 \mathrm{SV}$ automatic microtome (Microm, Walldorf, Germany). Histological images were acquired with an Axio Imager.A2 light microscope (Zeiss, Germany), equipped with a DC500 digital camera (Leica, Germany).

\section{Additional Files}

\begin{abstract}
Additional file 1: Figure S1. Histochemical localization of GUS activity in anthers of $p_{P 5 C S 1}: G U S$ and $p_{P 5 C S 2}$ GUS transgenic Arabidopsis plants. Inflorescences of $p_{\text {P5CS1: }}$ GUS (A-C) and $p_{\text {P5CS2: }}$ GUS (D-F) transgenic plants were infiltrated with X-Gluc solution, stained overnight, fixed and cleared for microscopic analysis. Each panel shows a whole-mount anther at stage 12/13 from an independent transgenic line. GUS activity was exclusively detected in pollen grains. Scale bars are $50 \mu \mathrm{m}$ in $\mathrm{A}, \mathrm{B}, \mathrm{D}, \mathrm{E}$ and $25 \mu \mathrm{m}$ in $\mathrm{C}$ and F. (PDF $2779 \mathrm{~kb}$ )

Additional file 2: Figure S2. Predicted cis-regulatory elements in the promoters of P5CS1 and P5CS2. Schematic map outlining the main putative binding sites for transcription factors derived from a PlantPAN2 (http://plantpan2.itps.ncku.edu.tw) and PLACE (http://www.dna.affrc.go.jp/ PLACE/) in silico analysis of P5CS1 (At2939800) and P5CS2 (At3955610) promoters. The promoter analysis was carried out on 2932 bp and 2097 bp upstream of the start codons of either P5CS1 or P5CS2, respectively. Putative cis-regulatory elements corresponding to binding motifs of transcription factors related to pollen development and fertility (SBP, bHLH, WRKY; GO terms "associated with pollen development" [GO:0009555], "pollen tube growth" [GO:0009860], "anther development" [GO:00048643] and "double fertilization forming a zygote and endosperm" [GO:0009567]) are significantly enriched and highlighted in red. (PDF $1149 \mathrm{~kb}$ )
\end{abstract}

Additional file 3: Figure S3. Histochemical localization of GUS activity

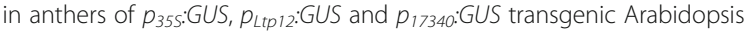
plants. Inflorescences of $p_{355}: G U S(\mathbf{A}, \mathbf{B}), p_{\text {Ltp } 12}: G U S(\mathbf{C}, \mathbf{D})$ and $p_{17340}: G U S$ $(\mathbf{E}, \mathrm{F})$ transgenic plants were infiltrated with $X$-Gluc solution, stained overnight, fixed and cleared for microscopic analysis. Each panel shows a whole-mount anther at stage 12/13 (A,B,E,F) or stage $9 / 10(\mathbf{C}, \mathbf{D})$ from an independent transgenic line. GUS activity was detected in the filaments and vascular tissues of $p_{35}: G U S$ anthers but not in pollen grains. The

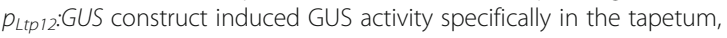
whereas GUS activity in $p_{17340}$ GUS transgenic anthers was almost exclusively detected in pollen grains. Scale bars are 50 m. (PDF 2798 kb)

Additional file 4: Table S1. Overview of pollen morphology and fertility data. (PDF $22 \mathrm{~kb}$ )

Additional file 5: Table S2. Primers used in this study (PDF 35 kb)

\section{Abbreviations}

CaMV35S: Cauliflower mosaic virus 355 transcript; CATMA: Complete arabidopsis transcriptome microarray; GUS: $\beta$-glucuronidase; LHT: Lysine histidine transporter; Ltp 12: Lipid transfer protein 12; P5C: $\Delta^{1}$-pyrroline-5carboxylate; P5CR: P5C reductase; P5CS: P5C synthetase; ProT: Proline transporter; X-Gluc: 5-bromo-4-chloro-3-indolyl- $\beta$-D-glucuronide.

\section{Acknowledgments}

We thank Dr. Valentina Cecchetti for helpful technical suggestions. We thank Rosi Miller, Silvia Kuhn and the gardener team of the University of Konstanz for help in genotyping and plant care.

\section{Funding}

This work was partially supported by research grants from Sapienza Università to MT, from the Italian Ministry of Education, University and Research (Progetti di Ricerca di Interesse Nazionale) to PC and from the University of Konstanz to DF.

\section{Availability of data and materials}

All mutants and constructs generated in this study are available from the corresponding authors on reasonable request.

\section{Authors' contributions}

The experiments were conceived, designed and carried out by MT, DF, and RM. Some molecular and genetic experiments were carried out by MB and AE. Biochemical analyses were designed and carried out by LM. The paper was written by MT, DF and PC. All authors have read and approved the final manuscript. 


\section{Ethics approval and consent to participate}

Seeds of Arabidopsis wildtype (Col-0) and T-DNA insertion lines were obtained from the Nottingham Arabidopsis Stock Centre (NASC) and were cultivated and genetically modified in the laboratories of M. Trovato at Sapienza University or D. Funck at the University of Konstanz in compliance with institutional, national, or international guidelines and legislation.

\section{Consent for publication}

Not applicable.

\section{Competing interests}

Prof. Paolo Costantino is an editorial board member of BMC Plant Biology, but was not involved in the editorial assessment of this manuscript. The other authors declare that they have no competing interests.

\section{Publisher's Note}

Springer Nature remains neutral with regard to jurisdictional claims in published maps and institutional affiliations.

\section{Author details}

'Department of Biology and Biotechnology, Sapienza University of Rome, P.le A. Moro 5, 00185 Rome, Italy. ${ }^{2}$ Department of Bioscience, University of Milan, Milan, Italy. ${ }^{3}$ Department of Biochemical Sciences, Sapienza University of Rome, Rome, Italy. ${ }^{4}$ Department of Biology, University of Konstanz, Universitätsstraße 10, 78464 Konstanz, Germany.

Received: 9 August 2018 Accepted: 23 November 2018 Published online: 17 December 2018

\section{References}

1. Biancucci M, Mattioli R, Forlani G, Funck D, Costantino P, Trovato M. Role of proline and GABA in sexual reproduction of angiosperms. Front Plant Sci. 2015;6:680.

2. Funck D, Winter G, Baumgarten L, Forlani G. Requirement of proline synthesis during Arabidopsis reproductive development. BMC Plant Biol. 2012;12:191.

3. Mattioli R, Biancucci M, Lonoce C, Costantino P, Trovato M. Proline is required for male gametophyte development in Arabidopsis. BMC Plant Biol. 2012;12:236

4. Chiang HH, Dandekar AM. Regulation of proline accumulation in Arabidopsis thaliana $(\mathrm{L})$ Heynh during development and in response to desiccation. Plant Cell Environ. 1995;18(11):1280-90.

5. Khoo U, Stinson HT. Free amino acid differences between cytoplasmic male sterile and normal fertile anthers. Proc Natl Acad Sci U S A. 1957;43(7):603-7.

6. Krogaard H, Andersen AS. Free amino-acids of Nicotiana-Alata anthers during development Invivo. Physiol Plant. 1983;57(4):527-31.

7. Lansac AR, Sullivan CY, Johnson BE. Accumulation of free proline in sorghum (Sorghum bicolor) pollen. Can J Bot/Rev Can Bot. 1996;74(1):40-5.

8. Mattioli R, Falasca G, Sabatini S, Altamura MM, Costantino P, Trovato M. The proline biosynthetic genes P5CS1 and P5CS2 play overlapping roles in Arabidopsis flower transition but not in embryo development. Physiol Plant. 2009;137(1):72-85.

9. Mattioli R, Marchese D, D'Angeli S, Altamura MM, Costantino P, Trovato M. Modulation of intracellular proline levels affects flowering time and inflorescence architecture in Arabidopsis. Plant Mol Biol. 2008;66(3):277-88.

10. Schwacke R, Grallath S, Breitkreuz KE, Stransky E, Stransky H, Frommer WB, Rentsch D. LeProT1, a transporter for proline, glycine betaine, and gammaamino butyric acid in tomato pollen. Plant Cell. 1999;11(3):377-92.

11. Mestichelli LJ, Gupta RN, Spenser ID. The biosynthetic route from ornithine to proline. J Biol Chem. 1979;254(3):640-7.

12. Roosens $\mathrm{NH}$, Thu TT, Iskandar HM, Jacobs M. Isolation of the ornithine-deltaaminotransferase cDNA and effect of salt stress on its expression in Arabidopsis thaliana. Plant Physiol. 1998;117(1):263-71.

13. Funck D, Stadelhofer B, Koch W. Ornithine- $\delta$-aminotransferase is essential for arginine catabolism but not for proline biosynthesis. BMC Plant Biol. 2008:8:40

14. Strizhov N, Ábrahám E, Ökrész L, Blickling S, Zilberstein A, Schell J, Koncz C, Szabados L. Differential expression of two P5CS genes controlling proline accumulation during salt-stress requires $A B A$ and is regulated by $A B A 1, A B \mid 1$ and AXR2 in Arabidopsis. Plant J. 1997;12(3):557-69.
15. Székely G, Ábrahám E, Cséplő A, Rigó G, Zsigmond L, Csiszár J, Ayaydin F, Strizhov N, Jásik J, Schmelzer E, et al. Duplicated P5CS genes of Arabidopsis play distinct roles in stress regulation and developmental control of proline biosynthesis. Plant J. 2008;53(1):11-28.

16. Bowman JL. Arabidopsis : an atlas of morphology and development. New York: Springer; 1994.

17. Sager R, Lee J-Y. Plasmodesmata in integrated cell signalling: insights from development and environmental signals and stresses. J Exp Bot. 2014;65(22): 6337-58.

18. Mariani C, De Beuckeleer M, Truettner J, Leemans J, Goldberg RB. Induction of male sterility in plants by a chimaeric ribonuclease gene. Nature. 1990; 347:737.

19. Yang SL, Xiea LF, Mao HZ, Puah CS, Yang WC, Jiang LX, Sundaresan V, Ye D. TAPETUM DETERMINANT1 is required for cell specialization in the Arabidopsis anther. Plant Cell. 2003:15(12):2792-804.

20. Cecchetti V, Celebrin D, Napoli N, Ghelli R, Brunetti P, Costantino P, Cardarelli M. An auxin maximum in the middle layer controls stamen development and pollen maturation in Arabidopsis. New Phytol. 2017; 213(3):1194-207.

21. Al Mamun E, Cantrill LC, Overall RL, Sutton BG. Cellular organisation in meiotic and early post-meiotic rice anthers. Cell Biol Int. 2005;29(11):903-13.

22. Winter D, Vinegar B, Nahal H, Ammar R, Wilson GV, Provart NJ. An "electronic fluorescent pictograph" browser for exploring and analyzing large-scale biological data sets. PLoS One. 2007;2(8):e718.

23. Hruz T, Laule O, Szabo G, Wessendorp F, Bleuler S, Oertle L, Widmayer P, Gruissem W, Zimmermann P. Genevestigator v3: a reference expression database for the meta-analysis of transcriptomes. Adv Bioinforma. 2008; 2008:420747.

24. Allemeersch J, Durinck S, Vanderhaeghen R, Alard P, Maes R, Seeuws K, Bogaert T, Coddens K, Deschouwer K, Van Hummelen P, et al. Benchmarking the CATMA microarray. A novel tool for Arabidopsis transcriptome analysis. Plant Physiol. 2005;137(2):588-601.

25. Girousse C, Bournoville R, Bonnemain J-L. Water deficit-induced changes in concentrations in proline and some other amino acids in the phloem sap of alfalfa. Plant Physiol. 1996;111(1):109-13.

26. Mäkelä $P$, Peltonen-Sainio $P$, Jokinen $K$, Pehu E, Setälä H, Hinkkanen $R$, Somersalo S. Uptake and translocation of foliar-applied glycinebetaine in crop plants. Plant Sci. 1996;121(2):221-30

27. Lehmann S, Gumy C, Blatter E, Boeffel S, Fricke W, Rentsch D. In planta function of compatible solute transporters of the AtProT family. J Exp Bot. 2011;62(2):787-96.

28. Foster J, Lee YH, Tegeder M. Distinct expression of members of the LHT amino acid transporter family in flowers indicates specific roles in plant reproduction. Sex Plant Reprod. 2008;21(2):143-52.

29. Ábrahám E, Rigó G, Székely G, Nagy R, Koncz C, Szabados L. Lightdependent induction of proline biosynthesis by abscisic acid and salt stress is inhibited by brassinosteroid in Arabidopsis. Plant Mol Biol. 2003;51(3): 363-72.

30. Guan Y, Meng X, Khanna R, LaMontagne E, Liu Y, Zhang S. Phosphorylation of a WRKY transcription factor by MAPKs is required for pollen development and function in Arabidopsis. PLoS Genet. 2014;10(5):e1004384.

31. Lei $R$, Li X, Ma Z, Lv Y, Hu Y, Yu D. Arabidopsis WRKY2 and WRKY34 transcription factors interact with VQ20 protein to modulate pollen development and function. Plant J. 2017;91(6):962-76.

32. Wilkinson JE, Twell D, Lindsey K. Activities of CaMV 355 and nos promoters in pollen: implications for field release of transgenic plants. J Exp Bot. 1997; 48(307):265-75.

33. Ariizumi T, Amagai M, Shibata D, Hatakeyama K, Watanabe M, Toriyama K. Comparative study of promoter activity of three anther-specific genes encoding lipid transfer protein, xyloglucan endotransglucosylase/hydrolase and polygalacturonase in transgenic Arabidopsis thaliana. Plant Cell Rep. 2002;21(1):90-6.

34. Costa-Nunes JA. A novel Arabidopsis marker line that strongly labels uninucleate microspores and the subsequent male gametophyte development stages. Springerplus. 2013;2(1):237

35. Honys D, Twell D. Transcriptome analysis of haploid male gametophyte development in Arabidopsis. Genome Biol. 2004;5(11):R85.

36. Hesse M, Pacini E, Willemse M. The tapetum: cytology, function, biochemistry and evolution. Vienna: Springer; 2012.

37. Zhang HQ, Croes AF. Proline metabolism in pollen - degradation of proline during germination and early tube growth. Planta. 1983;159(1):46-9. 
38. Yoshiba Y, Nanjo T, Miura S, Yamaguchi-Shinozaki K, Shinozaki K. Stressresponsive and developmental regulation of Delta(1)-pyrroline-5-carboxylate synthetase 1 (P5CS1) gene expression in Arabidopsis thaliana. Biochem Biophys Res Commun. 1999;261(3):766-72.

39. Zou J, Song L, Zhang W, Wang Y, Ruan S, Wu WH. Comparative proteomic analysis of Arabidopsis mature pollen and germinated pollen. J Integr Plant Biol. 2009;51(5):438-55.

40. Holmes-Davis R, Tanaka CK, Vensel WH, Hurkman WJ, McCormick S. Proteome mapping of mature pollen of Arabidopsis thaliana. Proteomics. 2005;5(18):4864-84.

41. Noir S, Brautigam A, Colby T, Schmidt J, Panstruga R. A reference map of the Arabidopsis thaliana mature pollen proteome. Biochem Biophys Res Commun. 2005;337(4):1257-66.

42. Ischebeck T, Valledor L, Lyon D, Gingl S, Nagler M, Meijon M, Egelhofer V, Weckwerth W. Comprehensive cell-specific protein analysis in early and late pollen development from diploid microsporocytes to pollen tube growth. Mol Cell Proteomics. 2014;13(1):295-310.

43. Rotsch AH, Kopka J, Feussner I, Ischebeck T. Central metabolite and sterol profiling divides tobacco male gametophyte development and pollen tube growth into eight metabolic phases. Plant J. 2017;92(1):129-46.

44. Lehmann S, Funck D, Szabados L, Rentsch D. Proline metabolism and transport in plant development. Amino Acids. 2010;39(4):949-62.

45. Bernsdorff F, Döring A-C, Gruner K, Schuck S, Bräutigam A, Zeier J. Pipecolic acid orchestrates plant systemic acquired resistance and defense priming via salicylic acid-dependent and -independent pathways. Plant Cell. 2016; 28(1):102-29.

46. Hellmann H, Funck D, Rentsch D, Frommer WB. Hypersensitivity of an Arabidopsis sugar signaling mutant toward exogenous proline application. Plant Physiol. 2000;123(2):779-89

47. Návarová H, Bernsdorff F, Döring AC, Zeier J. Pipecolic acid, an endogenous mediator of defense amplification and priming, is a critical regulator of inducible plant immunity. Plant Cell. 2012;24(12):5123-41.

48. Wang G, Zhang J, Wang G, Fan X, Sun X, Qin H, Xu N, Zhong M, Qiao Z, Tang $Y$, et al. Proline responding 1 plays a critical role in regulating general protein synthesis and the cell cycle in maize. Plant Cell. 2014;26(6):2582-600.

49. Karimi M, Inze D, Depicker A. GATEWAY vectors for Agrobacterium-mediated plant transformation. Trends Plant Sci. 2002;7(5):193-5.

50. Earley KW, Haag JR, Pontes O, Opper K, Juehne T, Song K, Pikaard CS. Gateway-compatible vectors for plant functional genomics and proteomics. Plant J. 2006;45(4):616-29.

51. Clough SJ, Bent AF. Floral dip: a simplified method for Agrobacteriummediated transformation of Arabidopsis thaliana. Plant J. 1998;16(6):735-43.

52. Bates $L S$, Waldren RP, Teare ID. Rapid determination of free proline for water-stress studies. Plant Soil. 1973;39(1):205.

53. Schneider CA, Rasband WS, Eliceiri KW. NIH image to ImageJ: 25 years of image analysis. Nat Methods. 2012;9(7):671-5.

54. Cecchetti V, Brunetti P, Napoli N, Fattorini L, Altamura MM, Costantino P, Cardarelli M. ABCB1 and ABCB19 auxin transporters have synergistic effects on early and late Arabidopsis anther development. J Integr Plant Biol. 2015; 57(12):1089-98.

55. Francioso A, Fanelli S, Vigli D, Ricceri L, Cavallaro RA, Baseggio Conrado A, Fontana M, D'Erme M, Mosca L. HPLC determination of bioactive sulfur compounds, amino acids and biogenic amines in biological specimens. Adv Exp Med Biol. 2017:975:535-49.

56. Jefferson RA, Kavanagh TA, Bevan MW. GUS fusions: beta-glucuronidase as a sensitive and versatile gene fusion marker in higher plants. EMBO J. 1987; 6(13):3901-7.

57. Plegt L, Bino RJ. B-glucuronidase activity during development of the male gametophyte from transgenic and non-transgenic plants. Mol Gen Genet. 1989;216:321-7.

58. Hu CY, Chee PP, Chesney RH, Zhou JH, Miller PD, O'Brien WT. Intrinsic GUSlike activities in seed plants. Plant Cell Rep. 1990;9:1-15.

59. Nishihara M, Ito M, Tanaka I, Kyo M, Ono K, Irifune K, Morikawa H. Expression of the B-glucuronidase gene in pollen of lily (Lilium longiflorum), tobacco (Nicotiana tabacum), Nicotiana rustica, and Peony (Paeonia lactiflora) by particle bombardment. Plant Physiol. 1993;102:357-61.

\section{Ready to submit your research? Choose BMC and benefit from:}

- fast, convenient online submission

- thorough peer review by experienced researchers in your field

- rapid publication on acceptance

- support for research data, including large and complex data types

- gold Open Access which fosters wider collaboration and increased citations

- maximum visibility for your research: over $100 \mathrm{M}$ website views per year

At BMC, research is always in progress.

Learn more biomedcentral.com/submissions 